\title{
Sleep quality changes in insomniacs and non-insomniacs after acute altitude exposure and its relationship with acute mountain sickness
}

This article was published in the following Dove Press journal:

Neuropsychiatric Disease and Treatment

31 July 2014

Number of times this article has been viewed

\section{Xu-gang Tang' \\ Ji-hang Zhang' \\ Xu-bin Gao' \\ Qian-ning $\mathrm{Li}^{2}$ \\ Jia-bei $\mathrm{Li}^{\prime}$ \\ Jie Yu' \\ Jun Qin' \\ Lan Huang'}

'Institute of Cardiovascular Diseases, ${ }^{2}$ Department of Neurology, Xinqiao

Hospital, Third Military Medical

University, Chongqing, People's

Republic of China
Correspondence: Lan Huang; Jun Qin Institute of Cardiovascular Diseases, Xinqiao Hospital,Third Military Medical University, 183 Xinqiao Main St, Shapingba District, Chongqing 400037, People's Republic of China

Tel/fax +86236875 560l

Email huanglan260@I26.com;

qinjunxq@।26.com
Objective: We aimed to observe the changes in subjective sleep quality among insomniacs and non-insomniacs after acute ascending to $3,700 \mathrm{~m}$ and its possible relationship with acute mountain sickness (AMS).

Methods: A total of 600 adult men were recruited. Subjects' subjective sleep quality was evaluated by the Athens Insomnia Scale. AMS was assessed using the Lake Louise scoring system. Arterial oxygen saturation was measured.

Results: Despite insomnia resolution in only a few subjects, the prevalence of insomnia among insomniacs remained stable at $90 \%$ after rapid ascent to 3,700 $\mathrm{m}$. However, among noninsomniacs, the prevalence of insomnia sharply increased to $32.13 \%$ in the first day of altitude exposure and progressively reduced to $4.26 \%$ by the 60 th day of altitude stay. Moreover, the prevalences of insomnia symptoms decreased more markedly from day 1 to day 60 at 3,700 m among non-insomniacs than among insomniacs. At 3,700 m, the prevalence of AMS among insomniacs was $79.01 \%, 60.49 \%$, and $32.10 \%$ on the first, third, and seventh days, respectively, which was significantly higher than that among non-insomniacs. Multivariate regression revealed that elevated Athens Insomnia Scale scores are an independent risk factor for AMS (adjusted odds ratio 1.388, 95\% confidence interval: 1.314-1.464, $P<0.001$ ), whereas high arterial oxygen saturation and long duration of altitude exposure are protective factors against AMS.

Conclusion: Our results suggest that the effect of high-altitude exposure on subjective sleep quality is more marked, but disappears more quickly, among non-insomniacs than among insomniacs, whereas AMS is especially common among insomniacs. Moreover, poor subjective sleep quality is a risk factor for AMS.

Keywords: Athens Insomnia Scale, arterial oxygen saturation, rapid ascent, sleep

\section{Introduction}

Subjective sleep complaints are very frequent among adolescents ${ }^{1}$ and adults ${ }^{2}$ in modern society, as well as among people exposed to high altitude. ${ }^{3}$ Thus, subjective insomnia is one of the primary components in the Lake Louise scoring system, which is a well-known list of diagnostic criteria for acute mountain sickness (AMS). ${ }^{4}$ AMS is a common syndrome that occurs in people, especially lowlanders, who are suddenly exposed to altitudes above $2,500 \mathrm{~m} .^{5}$ This syndrome is distinguished by headache, dizziness/lightheadedness, insomnia, gastrointestinal distress (nausea or vomiting), and weakness/fatigue. ${ }^{4}$ Although benign and self-limited, AMS may progress to a fatal outcome (fatal high-altitude pulmonary or cerebral edema) in a small number of people. ${ }^{6}$ As one important item of the Lake Louise diagnostic criteria for AMS, subjective insomnia is coded in accordance with one of four grades $(0=$ slept as well 
as usual, 1= did not sleep as well as usual, 2= woke up many times/had a poor night's sleep, or $3=$ could not sleep at all). ${ }^{4}$ At high altitudes, the sleep quality of the subjects cannot be adequately assessed by the Lake Louise diagnostic criteria for AMS alone. Among the Lake Louise diagnostic criteria for AMS, subjective insomnia is the second most $^{7}$ or even the most $^{8}$ common symptom. However, there are only a few studies on the effects of high-altitude exposure on sleep, all of which used small sample sizes and polysomnography. ${ }^{9-11}$ As such, no large-scale investigations of subjective sleep problems in insomniacs after altitude exposure using standardized scales have been published.

It is well known that exposure to high altitudes may negatively impact sleep quality. However, there is no published research reporting the different changes in subjective sleep quality among insomniacs and non-insomniacs after acute high-altitude exposure and the relationship between subjective sleep quality and AMS. Given the high stability of insomnia, ${ }^{12}$ we hypothesized that sleep quality would more markedly decrease among non-insomniacs than among insomniacs upon acute altitude exposure, and that poor sleep quality would correlate with AMS. Therefore, the aims of this study were to observe the changes in subjective sleep quality among insomniacs and non-insomniacs after acute ascending to $3,700 \mathrm{~m}$ and its possible relationship with AMS.

\section{Methods \\ Subjects and design}

In our study, all participants were lowlanders, and individuals with cardiopulmonary disease and those currently using some medications (eg, vitamin supplements, cold medicines, hypnotics, or herbal remedies) were excluded. Prior to the study, written informed consent, approved by the Ethics Review Board of the Xinqiao Hospital, Chongqing, People's Republic of China (decision number: 2012015), was obtained from each participant. The study protocol was registered with the Chinese Clinical Trial Registry (number ChiCTRRCS-12002232). Additionally, our study conformed to the principles outlined in the Declaration of Helsinki.

This study was conducted at $500 \mathrm{~m}$ (Chengdu, Sichuan Province) and 3,700 $\mathrm{m}$ (Lhasa, Tibet) from June 8 through August 25, 2012. The temperature ranged from $15^{\circ} \mathrm{C}$ to $28^{\circ} \mathrm{C}$ in the camp during the day. About 7 days before ascent, all participants underwent a systematic survey and a routine physical examination (chest X-ray, electrocardiogram, echocardiogram, blood pressure, heart rate [HR], and arterial oxygen saturation $\left.\left[\mathrm{SaO}_{2}\right]\right)$. Additionally, the participants' subjective sleep habits were assessed using the Athens
Insomnia Scale (AIS). ${ }^{13}$ The demographic characteristics (eg, age, race, education, drinking and smoking habits) of the participants were recorded in the questionnaire. All participants ascended from $500 \mathrm{~m}$ to $3,700 \mathrm{~m}$ by air in 2 hours; because of the limited logistics support, they did so in groups, with a 1-day interval between ascensions. After ascending to 3,700 $\mathrm{m}$, the participants completed the AIS questionnaire on the second, fourth, eighth, 15th, and 61st mornings, and the participants completed the Lake Louise AMS questionnaire on the second, fourth, and eighth mornings, and their $\mathrm{HR}$ and $\mathrm{SaO}_{2}$ were measured. All participants had the same daily regimen and refrained from vigorous exercises during the first 7 days of altitude exposure. All participants were given the same diet in the camp, and tea, coffee, and alcohol were forbidden. After 7 days' acclimatization at 3,700 m, the participants started to work as usual.

\section{Sleep quality assessment}

Because our field study had a large sample size and a short period of time for completion, we selected the concise AIS for the assessment of subjective sleep quality. The AIS has proven excellent reliability and validity, ${ }^{13}$ and is both quick and easy to administer. Thus, the AIS is considered an excellent tool in both clinical and study settings. ${ }^{14}$

The AIS questionnaire is composed of eight items: ${ }^{13}$ 1) difficulty in sleep induction; 2) arousal during the night; 3) awakening in the early morning; 4) total sleep duration; 5) total sleep quality; 6) decreased sense of well-being in the daytime; 7) decreased functioning in the daytime; and 8) daytime sleepiness. All items are rated on a 4-point Likert scale, from 0 (no problem) to 3 (very severe problem). The AIS is intended to record subjective sleep problems of the subjects that occurred $\geq$ three times/week during the preceding month. A higher AIS score (AISS) indicates poorer sleep quality, and insomnia has been defined as subjects with an AISS $\geq 6 .{ }^{15}$ Consequently, the participants were divided into an insomnia group and a non-insomnia group using the plain AIS results.

\section{AMS assessment}

In our study, on the first, third, and seventh days of high-altitude stay, in the mornings, all participants completed the Lake Louise AMS questionnaire, ${ }^{4}$ which contains the following five items: headache, dizziness/lightheadedness, insomnia, gastrointestinal distress, and fatigue/weakness. All items are rated on a 4-point Likert scale, from 0 (no symptoms) to 3 (severe symptoms). AMS was diagnosed when the subjects suffered from a headache and at least one of the other symptoms and had a total Lake Louise score (LLS) $\geq 3$. 


\section{Physiological parameters}

After the participants completed the Lake Louise AMS questionnaire and had a 15-minute rest in a comfortable chair, their blood pressure, $\mathrm{HR}$, and $\mathrm{SaO}_{2}$ values were measured. Their $\mathrm{HR}$ and $\mathrm{SaO}_{2}$ were measured using a finger-pulse oximeter (Onyx ${ }^{\circledR}$ 9500; Nonin Medical, Inc., Plymouth, MN, USA). All subjects put their hands into their pants pockets to sufficiently pre-warm the fingers before the measurements. Three consecutive measurements were taken for all parameters, with the mean of the three measurements used in the analysis.

\section{Statistical analyses}

The results are shown as the means \pm standard deviations or $\mathrm{n}(\%)$, as appropriate, and were obtained using SPSS software (v 13.0; SPSS Inc., Chicago, IL, USA). All tests were twotailed, and $P<0.05$ was considered significant. Mean values were compared using Student's $t$-test. To assess frequencies, the chi-squared test was used. The Mann-Whitney $U$ test was used to compare the non-normally distributed data. An analysis of variance for two-way repeated measures was used to determine the changes in $\mathrm{HR}$ and $\mathrm{SaO}_{2}$. Tukey's post hoc test was used for group comparisons. Relationships between LLS and age, body mass index, and other variables were determined using the Spearman rank test. Before the multivariate analysis, odds ratios with $95 \%$ confidence intervals were determined using logistic regression for the associations between AMS and risk factors. After univariate analysis, factors with $P$-values $\leq 0.10$ were entered into multivariate logistic regression analysis. The backward stepwise likelihood ratio test was used to determine the independent factors associated with AMS.

\section{Results}

\section{Subjects}

Before ascending, 625 participants completed the questionnaires and physical examination. Seven Tibetans were excluded because the sample size for this group was too small, and 18 other subjects were excluded for cardiac disease, medication use, and other reasons (eg, incomplete data, would not wish to join the study, etc). Table 1 shows the comparison of demographic characteristics of the insomnia and non-insomnia groups. At 3,700 m, data from 589 (insomnia group: 103 subjects), 567 (insomnia group: 99 subjects), 550 (insomnia group: 97 subjects), 450 (insomnia group: 89 subjects), and 386 (insomnia group: 81 subjects) participants were collected after 1, 3, 7, 14, and 60 days of exposure, respectively. On the first day of altitude exposure, five subjects were excluded from the study because of severe
Table I Demographic characteristics of the participants

\begin{tabular}{|c|c|c|c|}
\hline & $\begin{array}{l}\text { Insomnia } \\
\text { group }(n=8 I)\end{array}$ & $\begin{array}{l}\text { Non-insomnia } \\
\text { group }(n=305)\end{array}$ & $P$-value \\
\hline Age, years & $25.28 \pm 4.82$ & $23.17 \pm 4.21$ & $<0.001$ \\
\hline Weight, kg & $64.45 \pm 8.38$ & $64.37 \pm 7.41$ & $>0.05$ \\
\hline BMI, $\mathrm{kg} / \mathrm{m}^{2}$ & $22.52 \pm 2.8 \mathrm{I}$ & $21.91 \pm 2.19$ & $>0.05$ \\
\hline Smoking, yes & $50(61.73)$ & $168(55.08)$ & $>0.05$ \\
\hline Drinking, yes & II (13.58) & $36(11.80)$ & $>0.05$ \\
\hline Race & & & $>0.05$ \\
\hline Chinese Han & 7I (87.65) & $262(85.90)$ & \\
\hline Others & $10(12.35)$ & $43(14.10)$ & \\
\hline Education & & & $>0.05$ \\
\hline Junior school & $22(27.16)$ & $76(24.92)$ & \\
\hline High school & $36(44.44)$ & I 49 (48.85) & \\
\hline College & $23(28.40)$ & $80(26.23)$ & \\
\hline
\end{tabular}

Note: Data are shown as the mean \pm standard deviation or $\mathrm{n}(\%)$. Abbreviation: BMI, body mass index.

headache. Some participants missed the measurements because of work, and some participants left the camp and went down to the plains, which were the main reasons for the reduction of the number of data points in the present study. Additionally, one subject developed high-altitude pulmonary edema during the first night at high altitude and was excluded from the present study. Therefore, we analyzed data obtained from the remaining 386 subjects. The participants ranged from 17 to 45 years of age $(25 \% \leq 20$ years, $45 \% 21-25$ years, 24\% 26-30 years, and 6\% 31-45 years).

\section{Sleep quality}

On the first night of altitude exposure, the AISSs markedly increased when compared to those observed before ascent in each group, and gradually subsided during further exposure (Figure 1A). The decrease of AISSs in the non-insomnia group was more obvious. After 1 week of high-altitude stay, the AISSs in each group were still higher than they were before ascent, and this difference reached significance in the noninsomnia group. Similarly, in the first days of altitude exposure, the prevalence of insomnia in the non-insomnia group was significantly higher than it was before ascent (Figure 1B). After 2 months of acclimatization at 3,700 $\mathrm{m}$, the prevalence of insomnia in the non-insomnia group had decreased to a very low level $(4.26 \%)$. Notably, there were few subjects with an AISS $=6$ in the insomnia group who did not meet the AIS criterion for insomnia after arrival at high altitude, and the prevalence of insomnia in the insomnia group was stable at a high level (Figure 1B), even after 2 months of acclimatization at $3,700 \mathrm{~m}$. These data show that sleep quality altered more markedly, and improved more quickly, among non-insomniacs than among insomniacs after altitude exposure. 
A

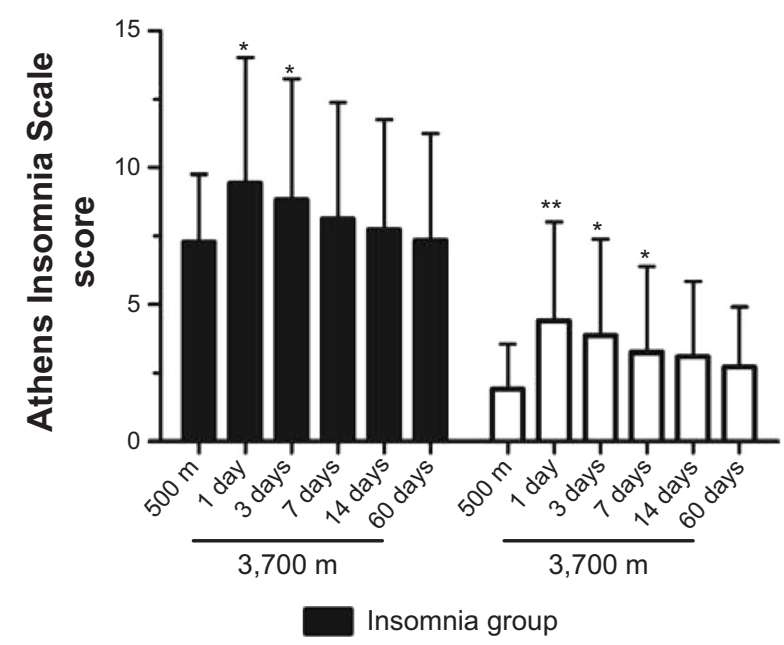

B

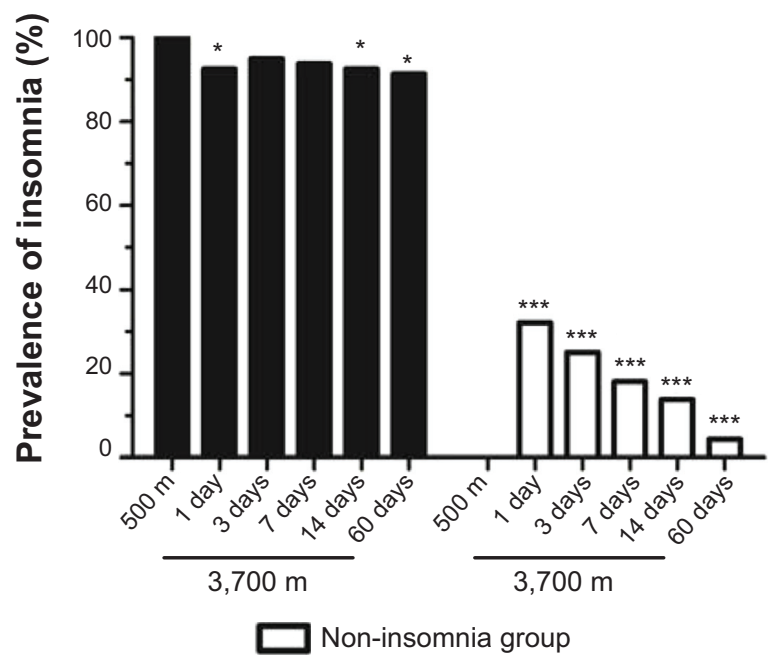

Figure I Changes in the Athens Insomnia Scale ${ }^{13}$ score $(\mathbf{A})$ and prevalence of insomnia $(\mathbf{B})$ among insomniacs and non-insomniacs after arrival at high altitude. Notes: Compared to the values obtained at $500 \mathrm{~m}$ in the same group: $* P<0.05, * * P<0.01, * * * P<0.001$.

Figure 2 shows the prevalence of symptoms of insomnia (AIS items) in the insomnia group and the non-insomnia group before and after high-altitude exposure. On the first night of altitude exposure, the prevalence of insomnia symptoms among the non-insomniacs sharply increased when compared to those observed before ascent. The most common symptom of insomnia in the non-insomnia group was arousal during the night, followed by difficulty in sleep induction and daytime sleepiness. After 2 months of high-altitude stay, the prevalences of insomnia symptoms decreased markedly from day 1 to day 60 at 3,700 $\mathrm{m}$ in the non-insomnia group, but were still significantly higher than they were before ascent. However, relatively small changes in the prevalence of insomnia symptoms in the insomnia group were observed. This may indicate that sleep quality improves more markedly in people with non-insomnia than in people with insomnia.

\section{AMS}

Figure 3 shows the LLSs and prevalence of AMS among the insomniacs and non-insomniacs after altitude exposure at $3,700 \mathrm{~m}$. In both groups, the LLSs reached the highest values after 1 day of 3,700 $\mathrm{m}$ exposure and then dropped markedly on the third and seventh days. Furthermore, the LLSs in the non-insomnia group were significantly lower than those in the insomnia group at different observation time points (Figure 3A).

The AMS prevalence in the insomnia group decreased sharply, from $79.01 \%$ to $60.49 \%$, on the third day and further decreased to $32.10 \%$ on the seventh day (Figure 3B). The same tendency was observed in the non-insomnia group, with the decrease of AMS prevalence in the non-insomnia group greater than that in the insomnia group. Moreover, the prevalence of AMS in the insomnia group was significantly higher than that in the non-insomnia group. Additionally, the prevalence of AMS in the whole group was $60.88 \%, 34.20 \%$, and $16.84 \%$ on the first, third, and seventh days, respectively.

Figure 4 shows the prevalence of AMS symptoms among the insomniacs and non-insomniacs at $3,700 \mathrm{~m}$. In the whole group, on the first day of altitude exposure, the most common symptom of AMS was headache (72.28\%), followed by dizziness/lightheadedness (70.47\%), weakness/fatigue (69.69\%), insomnia (62.95\%), and, rarely, gastrointestinal symptoms (19.17\%).

In the insomnia group, the prevalence of AMS symptoms reached the highest level on the first day of altitude exposure and then decreased, remaining significantly higher than those in the non-insomnia group at any observation time. Notably, relatively little change in the prevalence of insomnia was observed in the insomnia group; by comparison, the change in the prevalence of insomnia was more marked in the noninsomnia group. Additionally, with an increasing duration of altitude exposure, insomnia, as measured by the Lake Louise scoring system, became the most prominent symptom and was ahead of the other symptoms in both groups by the third day at 3,700 $\mathrm{m}$ (Figure 4).

\section{Physiological parameters}

The changes in physiological parameters are shown in Table 2. The HR in both the insomnia and non-insomnia 
A

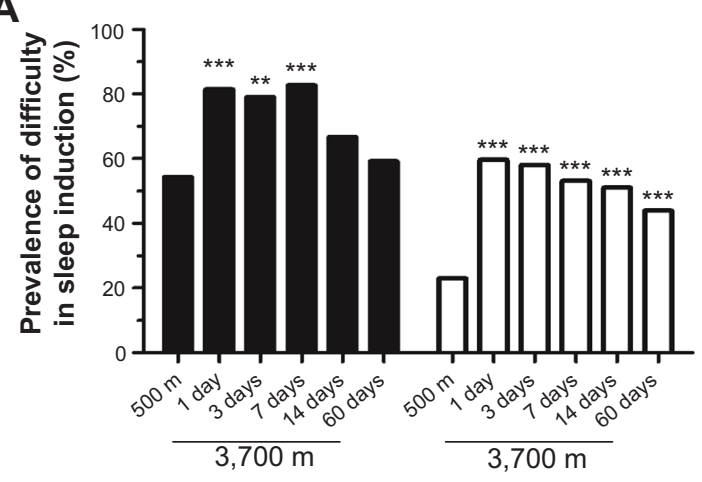

C

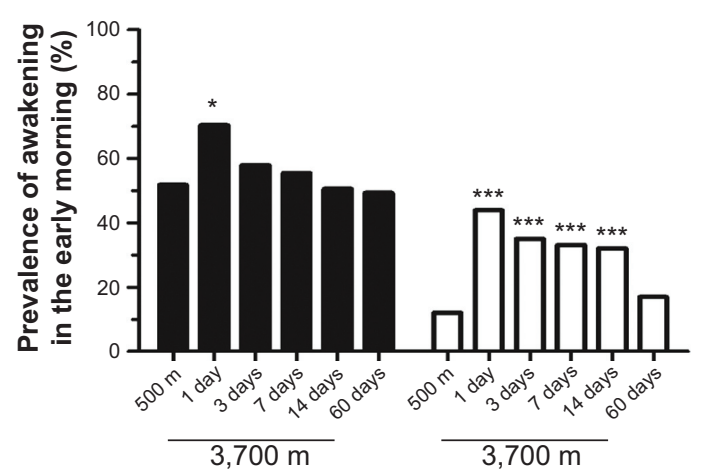

E

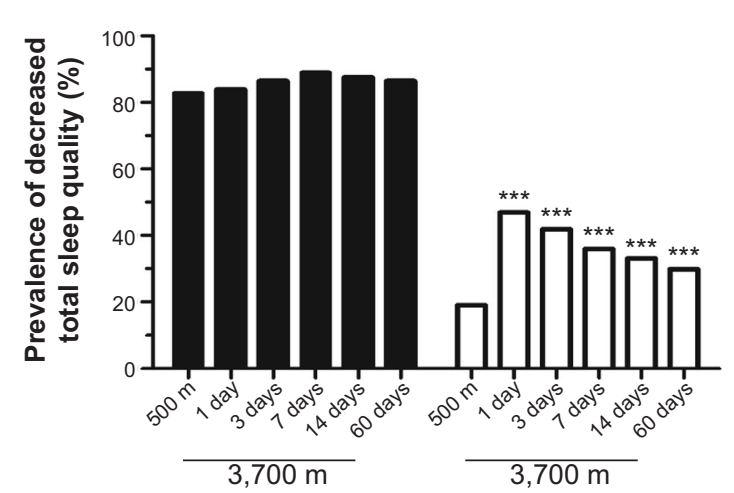

G

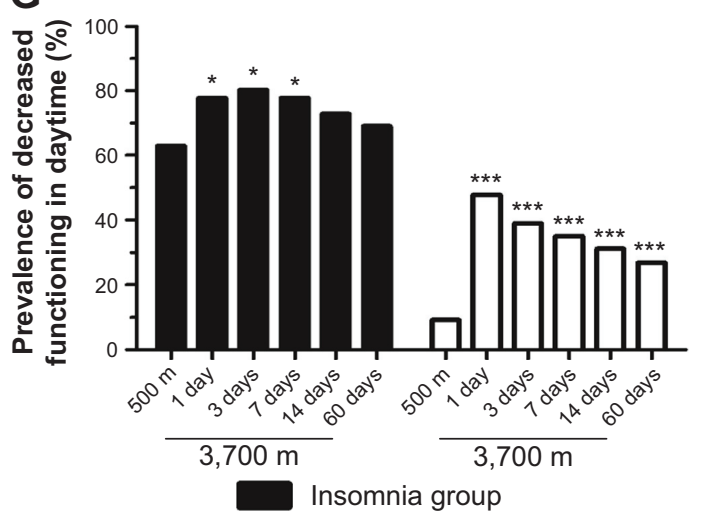

B

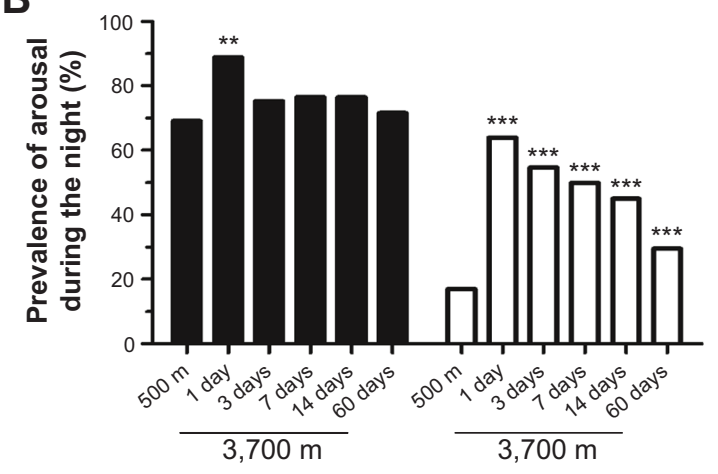

D

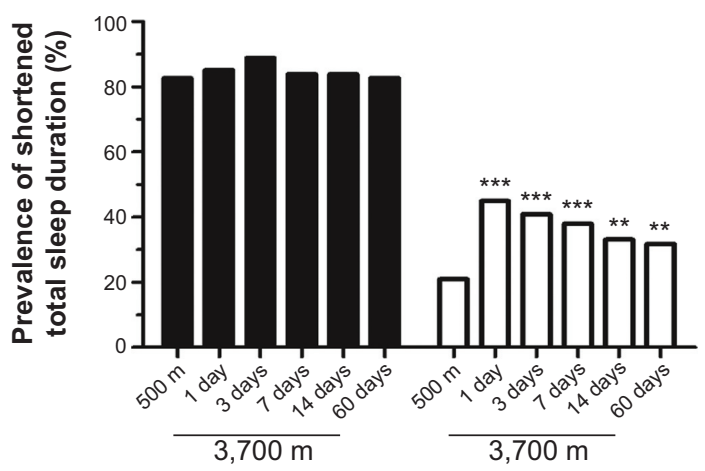

$\mathbf{F}$

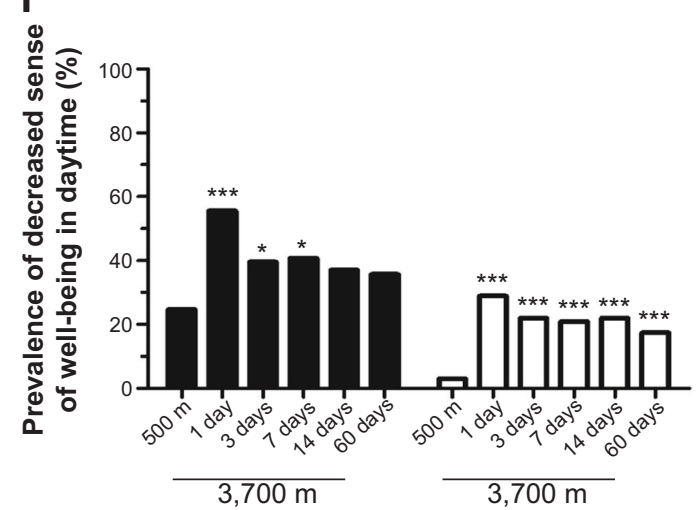

H

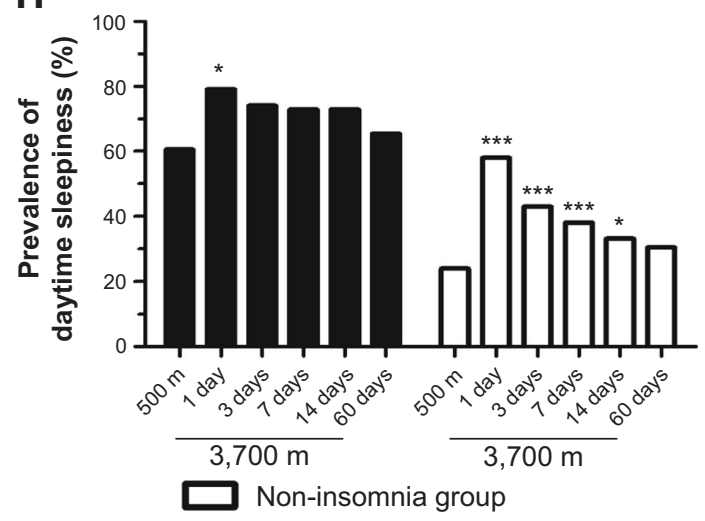

Figure 2 Changes in the prevalence of insomnia symptoms (Athens Insomnia Scale ${ }^{13}$ items) among insomniacs and non-insomniacs after arrival at high altitude. Notes: (A) Difficulty in sleep induction; (B) arousal during the night; (C) awakening in the early morning; (D) shortened total sleep duration; (E) decreased total sleep quality; (F) decreased sense of well-being in the daytime; $(\mathbf{G})$ decreased functioning in the daytime; $(\mathbf{H})$ daytime sleepiness. Compared to the values obtained at $500 \mathrm{~m}$ in the same group: $* p<0.05, * * P<0.01$, *** $P<0.001$. 
A

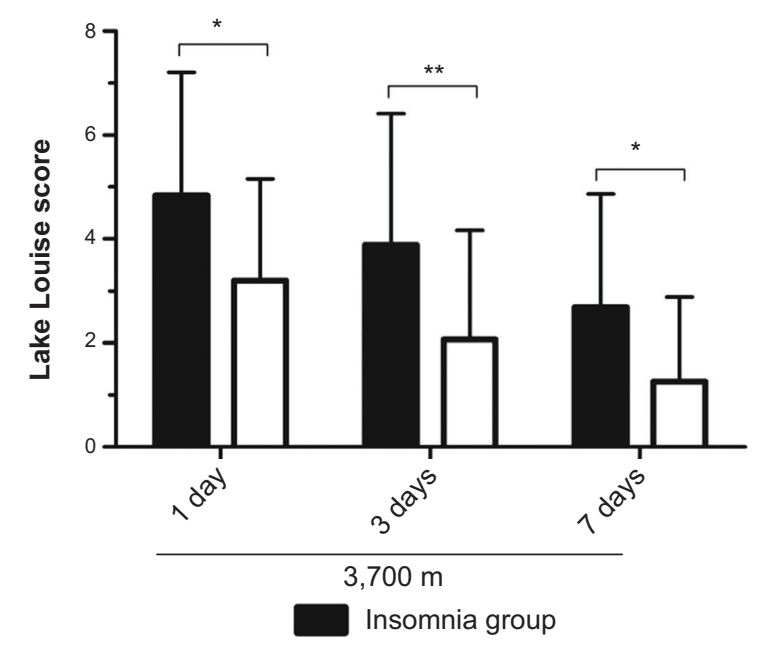

B

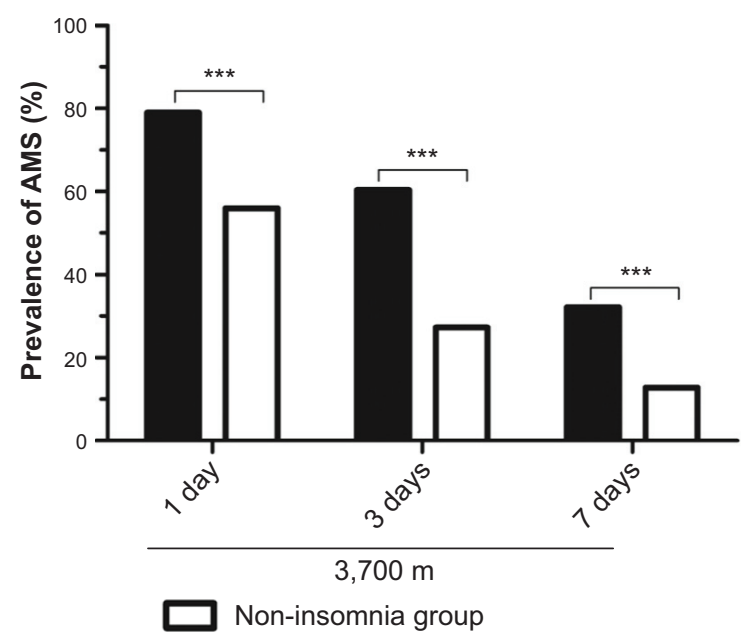

Figure 3 Comparisons of Lake Louise score ${ }^{4}(\mathbf{A})$ and the prevalence of AMS (B) between the insomnia and non-insomnia groups at 3,700 $\mathrm{m}$. Notes: $P$-values are indicated as: $* P<0.05, * * P<0.01$, $* * * P<0.001$.

Abbreviation: AMS, acute mountain sickness.

groups was elevated initially, then decreased with the duration of altitude exposure, but remained significantly higher compared to the level observed before ascent, even after 2 months of high-altitude stay. Furthermore, the HR in the insomnia group was significantly higher than that of the noninsomnia group on the first and third days at high altitude. On the contrary, the $\mathrm{SaO}_{2}$ in the insomnia group was slightly lower than in the non-insomnia group at high altitude.

\section{Correlational analysis}

To look for the risk factors for AMS, we first did the correlation analysis between LLSs and other factors. The results showed that age, HR, and AISSs were positively correlated with the LLSs $(\rho=0.096, P<0.05 ; \rho=0.092, P<0.01$; and $\rho=0.618$, $P<0.001$, respectively), whereas the $\mathrm{SaO}_{2}$ and duration of altitude exposure were negatively correlated with the LLSs ( $\rho=-0.155, P<0.01$ and $\rho=-0.332, P<0.001$, respectively). Note that there may be a spurious correlation between LLSs and AISSs, since the Lake Louise scoring system includes insomnia. Therefore, a statistical analysis of the correlation between LLSs and AISSs was carried out again, with the exclusion of the insomnia item from the Lake Louise scoring system. Our results showed that LLSs were still significantly and positively correlated with AISSs $(\rho=0.552, P<0.001)$. In addition, the AISSs before ascent were positively associated with the AISSs after altitude exposure $(\rho=0.343, P<0.001)$.

\section{Logistic regression analysis}

The results of a univariate logistic regression analysis showed that AMS was significantly positively associated with AISSs. In addition, AMS was significantly negatively associated with $\mathrm{SaO}_{2}$ and duration of altitude exposure (data not shown).

A multivariate logistic regression analysis that considered $\mathrm{SaO}_{2}$, duration of altitude exposure, and AISSs showed that all the parameters were significantly associated with AMS (Table 3).

\section{Discussion}

To date, our study, focusing on both insomniacs and noninsomniacs, is the first large-scale epidemiological investigation of the effects of acute altitude exposure on subjective sleep quality using the standardized AIS. The primary findings of our longitudinal study were that the sleep quality of insomniacs evaluated before ascent was slightly aggravated in the first days of altitude exposure, then displayed little change with the duration of altitude stay. However, the sleep quality of non-insomniacs was sharply aggravated after altitude exposure, then showed a continuously progressive improvement with the duration of altitude stay. Furthermore, high AISSs were a risk factor for AMS, whereas high $\mathrm{SaO}_{2}$ and long duration of altitude exposure were protective factors against AMS.

In the present study, approximately 600 participants were recruited. At $500 \mathrm{~m}$, the prevalence of subjective insomnia in the entire group was $20.98 \%$ when evaluated using the AIS, which is within the reported range $(2 \%-23 \%)$ for Chinese populations. ${ }^{16,17}$

At $3,700 \mathrm{~m}$, the prevalence of subjective insomnia in the non-insomnia group on the first, third, seventh, 14th, 
A

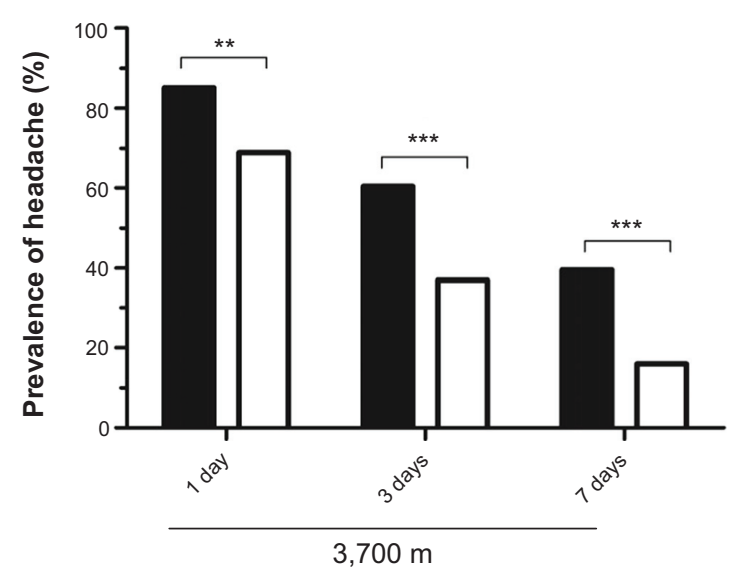

C

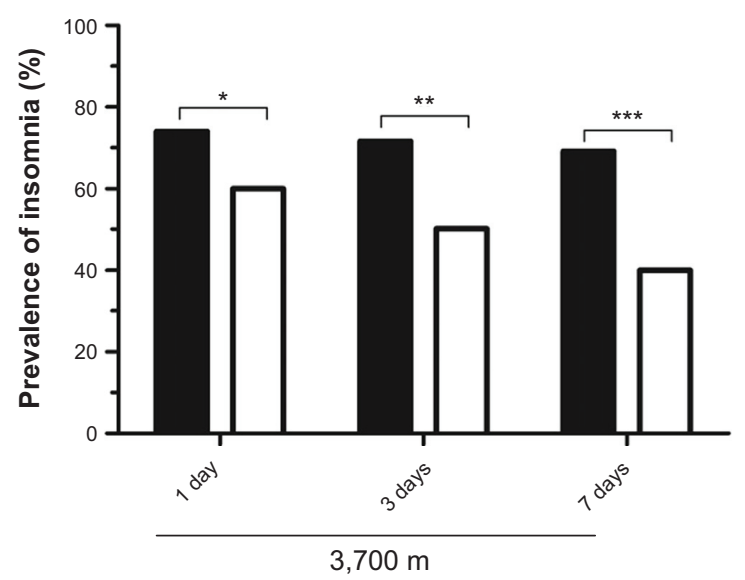

B

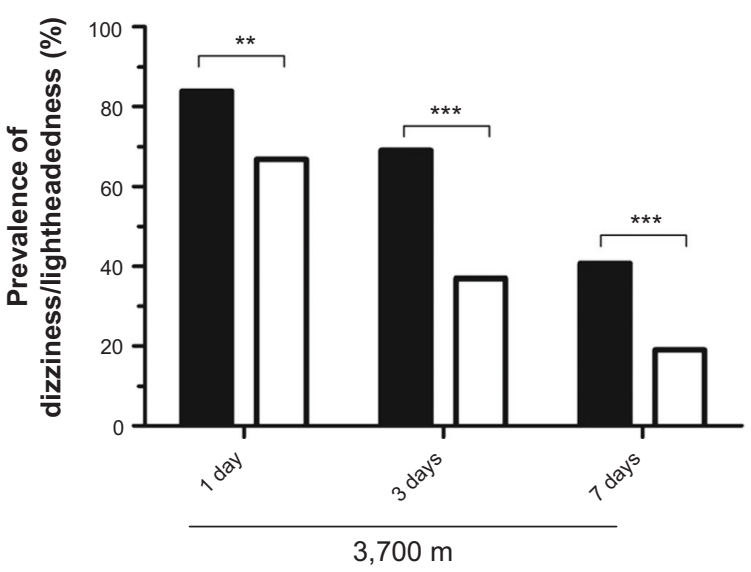

D

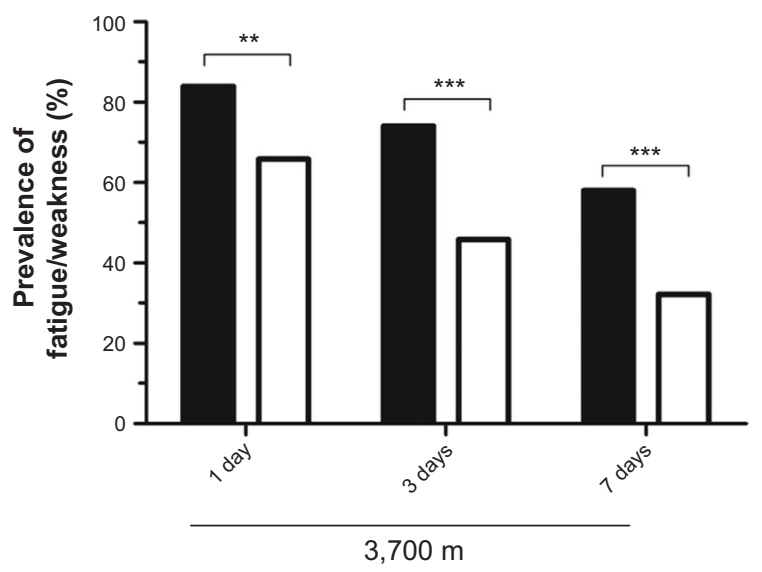

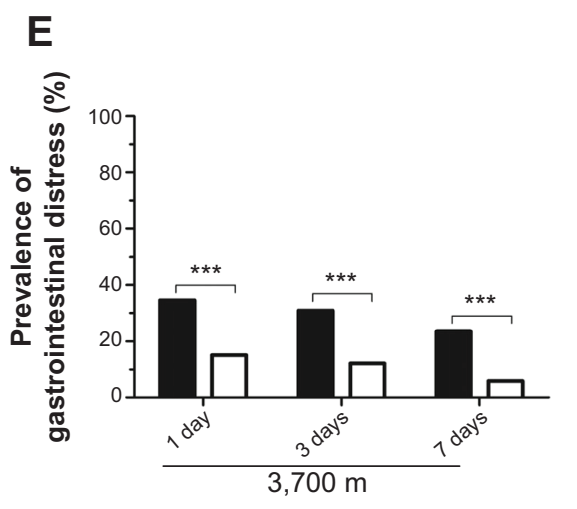

Insomnia group $\square$ Non-insomnia group

Figure 4 Comparisons of the prevalence of acute mountain sickness symptoms.

Notes: (A) Headache; (B) dizziness/lightheadedness; (C) insomnia; (D) fatigue/weakness; (E) gastrointestinal distress. Comparisons were performed with chi-squared tests: $* P<0.05$, ** $P<0.01$, *** $P<0.001$.

and 60 th nights was $32.13 \%, 24.92 \%, 18.03 \%, 13.77 \%$, and $4.26 \%$, respectively, according to the AIS (Figure 1B). Obviously, the rates in our study were lower than that reported by Szymczak et al (47\%, confirmed by the AIS), ${ }^{18}$ which may be partly due to the higher altitude (mean altitude 4,524 m) reached by the subjects in Szymczak et al's study. Studies ${ }^{9,18,19}$ have suggested that altitude hypoxia is a primary factor for the acute impairment of sleep upon altitude exposure. Thus, oxygen enrichment ${ }^{20}$ and altitude acclimatization $^{9,21}$ improve sleep quality at high altitude, but only partially. Therefore, the change in the prevalence of subjective insomnia in the non-insomnia group in the 
Table 2 Physiological parameters between the insomnia and non-insomnia groups

\begin{tabular}{|c|c|c|c|c|c|c|}
\hline & \multirow[t]{2}{*}{$500 \mathrm{~m}$} & \multicolumn{5}{|l|}{$3,700 \mathrm{~m}$} \\
\hline & & I day & 3 days & 7 days & 14 days & 60 days \\
\hline \multicolumn{7}{|l|}{$\mathrm{HR}$, bpm } \\
\hline Insomnia group & $65.77 \pm 8.98$ & $87.22 \pm 12.57^{*}$ & $86.29 \pm 10.34^{*}$ & $84.81 \pm 10.03 *$ & $82.11 \pm 9.45^{*}$ & $80.15 \pm 9.75 *$ \\
\hline Non-insomnia group & $66.50 \pm 8.76$ & $84.28 \pm 12.80^{*, \#}$ & $83.07 \pm 10.11^{*, \#}$ & $82.69 \pm 9.72 *$ & $80.85 \pm 9.33^{*}$ & $79.47 \pm 10.63^{*}$ \\
\hline \multicolumn{7}{|l|}{$\mathrm{SaO}_{2}, \%$} \\
\hline Insomnia group & $97.96 \pm 0.99$ & $88.37 \pm 3.18^{*}$ & $88.75 \pm 3.44 *$ & $89.58 \pm 3.45^{*}$ & $90.33 \pm 3.77^{*}$ & $90.74 \pm 3.32 *$ \\
\hline Non-insomnia group & $98.07 \pm 0.95$ & $89.32 \pm 3.11^{*}$ & $90.30 \pm 3.59 *$ & $90.83 \pm 3.53 *$ & $90.95 \pm 3.50 *$ & $91.21 \pm 3.58^{*}$ \\
\hline
\end{tabular}

Notes: Data are shown as the mean \pm standard deviation. ANOVA for repeated measures, compared to the values obtained at $500 \mathrm{~m}$ within group: $* \mathrm{P}<0.00 \mathrm{I}$; compared to the values of the insomnia group on the same day: ${ }^{\#} P<0.05$.

Abbreviations: ANOVA, analysis of variance; $\mathrm{HR}$, heart rate; $\mathrm{SaO}_{2}$, arterial oxygen saturation.

present study is supported by the literature. Remarkably, very few subjects with an AISS $=6$ before ascent in the insomnia group did not meet the AIS criterion for insomnia after arrival at high altitude, and the prevalence of subjective insomnia in this group remained at a high level (over 90\%) (Figure 1). This suggests that, for a small proportion of people, especially those with an AISS $=6$ before ascent, altitude exposure does not impair sleep quality. Additionally, because of the preexisting chronic sleep problems, the prevalence of subjective insomnia in an insomnia group is unlikely to fall as much as in the non-insomnia group with the duration of altitude exposure.

Notably, according to the Lake Louise AMS scoring system, subjective insomnia was the fourth most common symptom $(62.95 \%)$ in the entire group on the first day at $3,700 \mathrm{~m}$. However, it gradually became the most frequent symptom during the prolonged high-altitude stay in both the insomnia and non-insomnia groups (Figure 4). Remarkably, the prevalence of subjective insomnia (confirmed by the Lake Louise scoring system) in the insomnia group was consistently higher than in the non-insomnia group, but the decrease of subjective insomnia prevalence in the insomnia group was less than that in the non-insomnia group with the duration of high-altitude stay (Figure 4C). Furthermore, our data showed that high AISSs were a risk factor for AMS, as confirmed by a multivariate logistic regression analysis. These results reveal that the subjects with poor subjective sleep quality were more susceptible to AMS compared to those subjects with good subjective sleep quality.
After acute altitude exposure, all symptoms of subjective insomnia (AIS items) initially increased in both groups and decreased thereafter (Figure 2). Because this acute insomnia is mainly caused by hypoxia, ${ }^{9,18,19}$ the symptoms of subjective insomnia improved with acclimatization duration and were accompanied by an increase in $\mathrm{SaO}_{2}$. Moreover, the prevalences of subjective insomnia symptoms decreased more markedly from day 1 to day 60 at 3,700 $\mathrm{m}$ in the noninsomnia group than in the insomnia group. This may indicate that subjective sleep quality is affected by altitude hypoxia more markedly in non-insomniacs than in insomniacs.

Sleep fragmentation might be the primary factor for poor sleep at high altitude, ${ }^{22}$ which results in a sharply increased number of arousals among subjects. In our study, subjective arousals during the first night at $3,700 \mathrm{~m}$ were common in both groups (Figure 2B), which was consistent with the findings of Szymczak et al. ${ }^{18}$ Interestingly, after 2 months' stay at $3,700 \mathrm{~m}$, the prevalence of subjective nocturnal arousals in the insomnia group returned to almost the level before ascent, whereas that in the non-insomnia group did not. One possible reason may be that the prevalence of subjective arousals in the insomnia group consistently remained at a high level before ascent so that it was relatively easy for arousal prevalence to decrease after a slight increase.

Difficulty in sleep induction after altitude exposure was the second most frequent subjective insomnia symptom observed in the present study (Figure 2A), which was consistent with the findings of Szymczak et al's study ${ }^{18}$ conducted with the AIS.

Table 3 Multivariate logistic regression analysis of the risk factors for acute mountain sickness

\begin{tabular}{llllll}
\hline & $\beta$ & SE & Wald & P-value & OR (95\% CI) \\
\hline $\mathrm{AlSSs}^{13}$ & 0.336 & 0.020 & 227.481 & $<0.001$ & $1.388(1.314-1.464)$ \\
$\mathrm{SaO}_{2}$ & -0.057 & 0.018 & 7.476 & $<0.01$ & $0.931(0.894-0.971)$ \\
Duration of altitude exposure & -1.232 & 0.131 & 98.105 & $<0.001$ & $0.296(0.227-0.378)$ \\
\hline
\end{tabular}

Note: Dependent variable: acute mountain sickness.

Abbreviations: AISS, Athens Insomnia Scale score; $\mathrm{Cl}$, confidence interval; OR, odds ratio; $\mathrm{SaO}_{2}$, arterial oxygen saturation; $\mathrm{SE}$, standard error. 
In the present study, we observed a markedly increased prevalence of subjectively shortened total sleep duration in the non-insomnia group (Figure 2D). However, in Szymczak et al's study conducted using the AIS, ${ }^{18}$ the subjective total sleep duration of the subjects showed only a decreasing trend, partly because of the research setting and small sample (32 participants).

The prevalence of AMS has been shown in the literature to vary depending on the setting, from between $9 \%$ and $69 \%$ in the $\mathrm{Alps}^{23}$ to between $45 \%$ and $95 \%$ in Tibet. ${ }^{24}$ In our study, at $3,700 \mathrm{~m}$, the prevalence of AMS in the insomnia group was $79.01 \%, 60.49 \%$, and $32.10 \%$ on the first, third, and seventh days, respectively, which was significantly higher compared to prevalence in the non-insomnia group. With a longer duration of exposure, the prevalence of AMS decreased in both groups; however, the decrease of AMS prevalence in the non-insomnia group was greater than that in the insomnia group. One reason for this was the relatively higher $\mathrm{SaO}_{2}$ in the non-insomnia group; another may be the poor subjective sleep quality in the insomnia group. Our data showed that the prevalences of AMS symptoms in the insomnia group were significantly higher than those in the non-insomnia group (Figure 4), which directly resulted in the higher LLS in the former. Furthermore, elevated AISS were positively correlated with AMS.

Supported by previous study, ${ }^{25}$ our data revealed that the HR in both the insomnia and non-insomnia groups markedly increased on the first day at 3,700 $\mathrm{m}$ and subsided from the third day of high-altitude stay, but remained higher than the values obtained before ascent, even after 2 months of acclimatization. Compared to the non-insomnia group, significantly higher HR in the insomnia group may indicate that the degree of hypoxia in the insomnia group was more serious, which may be one reason for the higher prevalence of AMS in the insomnia group. The converse change in $\mathrm{SaO}_{2}$ was observed in both groups (Table 2).

High $\mathrm{SaO}_{2}$ and long duration of altitude exposure were protective factors against AMS in the present study (Table 3). Our $\mathrm{SaO}_{2}$ results are consistent with those of $\mathrm{Wu}$ et $\mathrm{al}^{7}$ but contrary to those of Chen et al. ${ }^{26}$ The ascent rate may have accounted for this discrepancy.

One of the main strengths of our study is that it was longitudinal in design. The other strengths are the relatively large sample size and the same altitude, ascent rate, and daily regimen. However, the present study has some limitations. First, all of the participants in the present study were young adult men, which may generate a bias of age or sex. Second, the dropout rate of the entire group was relatively high, reaching almost $25 \%$ and $36 \%$ on the last 2 days of observations, respectively. These limitations can be improved in future studies.

\section{Conclusion}

Our results suggest that the effect of high-altitude exposure on subjective sleep quality is more marked, but disappears more quickly, among non-insomniacs than among insomniacs, whereas AMS is especially common among the latter. Moreover, poor subjective sleep quality is a risk factor for AMS.

\section{Acknowledgments}

This study was supported by a grant from the Special Health Research Project, Ministry of Health of China (No 201002012). We thank all the participants, as well as all investigators, whose participation allowed the study to be conducted. We also thank the editors of American Journal Experts for the manuscript editing.

\section{Disclosure}

The authors report no conflicts of interest in this work.

\section{References}

1. Carotenuto M, Gallai B, Parisi L, Roccella M, Esposito M. Acupressure therapy for insomnia in adolescents: a polysomnographic study. Neuropsychiatr Dis Treat. 2013;9:157-162.

2. Fang J, Wheaton AG, Keenan NL, Greenlund KJ, Perry GS, Croft JB. Association of sleep duration and hypertension among US adults varies by age and sex. Am J Hypertens. 2012;25(3):335-341.

3. Imray C, Wright A, Subudhi A, Roach R. Acute mountain sickness: pathophysiology, prevention, and treatment. Prog Cardiovasc Dis. 2010;52(6):467-484.

4. Roach RC, Bartsch P, Hackett PH, Oelz O. The Lake Louise acute mountain sickness scoring system. In: Sutton JR, Houston CS, Coates G, editors. Hypoxia and Molecular Medicine. Burlington, VT: Queen City Press; 1993:272-274.

5. Bärtsch P, Swenson ER. Acute high-altitude illnesses. $N$ Engl J Med. 2013;368(24):2294-2302.

6. Netzer N, Strohl K, Faulhaber M, Gatterer H, Burtscher M. Hypoxiarelated altitude illnesses. J Travel Med. 2013;20(4):247-255.

7. Wu TY, Ding SQ, Liu JL, et al. Smoking, acute mountain sickness and altitude acclimatisation: a cohort study. Thorax. 2012;67(10): 914-919.

8. Jafarian S, Gorouhi F, Ghergherechi M, Lotfi J. Respiratory rate within the first hour of ascent predicts subsequent acute mountain sickness severity. Arch Iran Med. 2008;11(2):152-156.

9. Nussbaumer-Ochsner Y, Ursprung J, Siebenmann C, Maggiorini M, Bloch KE. Effect of short-term acclimatization to high altitude on sleep and nocturnal breathing. Sleep. 2012;35(3):419-423.

10. de Aquino Lemos V, Antunes HK, dos Santos RV, Lira FS, Tufik S, de Mello MT. High altitude exposure impairs sleep patterns, mood, and cognitive functions. Psychophysiology. 2012;49(9):1298-1306.

11. Reite M, Jackson D, Cahoon RL, Weil JV. Sleep physiology at high altitude. Electroencephalogr Clin Neurophysiol. 1975;38(5):463-471.

12. Buysse DJ, Angst J, Gamma A, Ajdacic V, Eich D, Rössler W. Prevalence, course, and comorbidity of insomnia and depression in young adults. Sleep. 2008;31(4):473-480. 
13. Soldatos CR, Dikeos DG, Paparrigopoulos TJ. Athens Insomnia Scale: validation of an instrument based on ICD-10 criteria. J Psychosom Res. 2000;48(6):555-560.

14. Gómez-Benito J, Ruiz C, Guilera G. A Spanish version of the Athens Insomnia Scale. Qual Life Res. 2011;20(6):931-937.

15. Soldatos CR, Dikeos DG, Paparrigopoulos TJ. The diagnostic validity of the Athens Insomnia Scale. J Psychosom Res. 2003;55(3):263-267.

16. Liu X, Zhou H. Sleep duration, insomnia and behavioral problems among Chinese adolescents. Psychiatry Res. 2002;111(1):75-85.

17. Chiu HF, Xiang YT, Dai J, et al. The prevalence of sleep problems and their socio-demographic and clinical correlates in young Chinese rural residents. Psychiatry Res. 2012;200(2-3):789-794.

18. Szymczak RK, Sitek EJ, Sławek JW, Basiński A, Siemiński M, Wieczorek D. Subjective sleep quality alterations at high altitude. Wilderness Environ Med. 2009;20(4):305-310.

19. Mizuno K, Asano K, Okudaira N. Sleep and respiration under acute hypobaric hypoxia. Jpn J Physiol. 1993;43(2):161-175.
20. Luks AM, van Melick H, Batarse RR, Powell FL, Grant I, West JB. Room oxygen enrichment improves sleep and subsequent day-time performance at high altitude. Respir Physiol. 1998;113(3):247-258.

21. Mortola JP, Seifert EL. Hypoxic depression of circadian rhythms in adult rats. J Appl Physiol (1985). 2000;88(2):365-368.

22. Weil JV. Sleep at high altitude. High Alt Med Biol. 2004;5:180-189.

23. Maggiorini M, Bühler B, Walter M, Oelz O. Prevalence of acute mountain sickness in the Swiss alps. BMJ. 1990;301(6756):853-855.

24. Wu TY, Ding SQ, Liu JL, et al. Ataxia: an early indicator in high altitude cerebral edema. High Alt Med Biol. 2006;7:275-280.

25. Selvamurthy W, Raju VR, Ranganathan S, Hegde KS, Ray US. Sleep patterns at an altitude of 3,500 metres. Int J Biometeorol. 1986;30(2): 123-135.

26. Chen HC, Lin WL, Wu JY, et al. Change in oxygen saturation does not predict acute mountain sickness on Jade Mountain. Wilderness Environ Med. 2012;23(2):122-127.
Neuropsychiatric Disease and Treatment

\section{Publish your work in this journal}

Neuropsychiatric Disease and Treatment is an international, peerreviewed journal of clinical therapeutics and pharmacology focusing on concise rapid reporting of clinical or pre-clinical studies on a range of neuropsychiatric and neurological disorders. This journal is indexed on PubMed Central, the 'PsycINFO' database and CAS,

\section{Dovepress}

and is the official journal of The International Neuropsychiatric Association (INA). The manuscript management system is completely online and includes a very quick and fair peer-review system, which is all easy to use. Visit http://www.dovepress.com/testimonials.php to read real quotes from published authors. 